\title{
Use of NRP1, a novel biomarker, along with VEGF-C, VEGFR-3, CCR7 and SEMA3E, to predict lymph node metastasis in squamous cell carcinoma of the tongue
}

\author{
HANI AL-SHAREEF ${ }^{1}$, SHIN-ICHIRO HIRAOKA ${ }^{1}$, NORIAKI TANAKA ${ }^{2}$, YOSUKE SHOGEN $^{1}$, \\ ATSUSHI-DOKSA LEE ${ }^{1}$, SANAM BAKHSHISHAYAN ${ }^{1}$ and MIKIHIKO KOGO ${ }^{1}$
}

${ }^{1}$ First Department of Oral and Maxillofacial Surgery, Graduate School of Dentistry, Osaka University, Osaka 565-0871, Japan;

${ }^{2}$ Department of Head and Neck Surgery, University of Texas MD Anderson Cancer Center, Houston, TX 77030, USA

Received March 31, 2016; Accepted August 5, 2016

DOI: 10.3892/or.2016.5116

\begin{abstract}
Lymph node (LN) metastasis has been suggested as a major prognostic factor for oral cancer. Knockdown of the growth factors and receptors involved in these metastatic mechanisms could significantly reduce LN metastasis and improve the survival of oral cancer patients after treatment. The present study, therefore, aimed to evaluate the expression levels of the following growth factors and receptors in squamous cell carcinoma (SCC) of the tongue: the vascular endothelial growth factor (VEGF)-C and VEGF-D, which bind to the cell surface tyrosine kinase receptor VEGF receptor-3 (VEGFR-3); C-C motif chemokine receptor 7 (CCR7); neuropilin (NRP)1 and NRP2; and semaphorin 3E (SEMA3E). Furthermore, we assessed microvessel density (MVD) and lymphatic vessel density (LVD) to demonstrate the correlation between these factors and regional LN metastasis, with respect to the clinicopathological features. Finally, we analyzed the correlation between these proteins and overall or disease-free survival, in order to demonstrate their prognostic value. Univariate analysis revealed a significant association between LN metastasis and the expression levels of VEGF-C, VEGFR-3, CCR7, NRP1, and SEMA3E, as well as LVD, in SCC cells. In contrast, multivariate analysis identified associations between LN metastasis and NRP1 expression, as well as between LN metastasis and LVD; however, no correlation was found between LN metastasis and the expression levels of the other proteins. The expression levels of VEGF-C, VEGFR-3, NRP1, and
\end{abstract}

Correspondence to: Dr Shin-ichiro Hiraoka, First Department of Oral and Maxillofacial Surgery, Graduate School of Dentistry, Osaka University, 1-8 Yamadaoka, Suita, Osaka 565-0871, Japan E-mail: hirashins2@gmail.com

Key words: squamous cell carcinoma, vascular endothelial growth factor-C, vascular endothelial growth factor-D, vascular endothelial growth factor receptor-3, C-C chemokine receptor type 7, semaphorin $3 \mathrm{E}$, lymphatic vessel density
SEMA3E, as well as LVD, were correlated with disease-free survival time. These results indicate that LN metastasis is associated with poor survival in SCC. This study suggests that NRP1 expression and LVD are independent factors that are likely to predict the risk of LN metastasis in SCC of the tongue, whereas the expression of VEGF-C, VEGFR-3, CCR7, and SEMA3E are non-independent predictive factors.

\section{Introduction}

Oral squamous cell carcinoma (SCC) is the most commonly identified cancer of the oral cavity and the head and neck region. More than $90 \%$ of the tumors occurring in the head and neck region are SCCs; these can grow in several locations of the mucosal lining, with relatively rare neoplasms arising in the minor salivary glands and soft tissues $(1,2)$.

Lymphatic metastasis is the most significant predictive factor for the survival of patients with oral SCC. Moreover, patients who suffer from recurrence of neck cancer, after initial treatment, frequently die due to uncontrolled neck disease and many other evaluated potential prognostic factors (3).

The vascular endothelial growth factor (VEGF) family including VEGF-A, VEGF-B, VEGF-C, VEGF-D, VEGF-E, and placental growth factor (PlGF), each acting through their respective VEGF tyrosine kinase receptors (VEGFR-1, VEGFR-2, and VEGFR-3), play significant roles in angiogenesis and lymphangiogenesis (4). Many studies have reported that the overexpression of VEGF-C or VEGF-D, acting through the tyrosine kinase receptor VEGF receptor-3 (VEGFR-3), promotes the process of lymph node (LN) metastasis through the regulation of cancer lymphangiogenesis (5). Moreover, VEGF-C and VEGF-D are associated with LN metastasis through intratumoral and peritumoral lymphatics in many types of cancers, including esophageal, gastric, colon, breast, thyroid, pancreas, prostate, and lung cancers (6).

Small pro-inflammatory 'chemotactic cytokines' are secreted proteins that are involved in the migration of activated hematopoietic cells (dendritic cells) to regional LNs (7). The chemokine superfamily is generally classified into four groups based on the cysteine motifs in the protein (CXC, CC, $\mathrm{CX} 3 \mathrm{C}$, and $\mathrm{C}$ ), and the involvement of these proteins in tumor 
metastasis has been determined (8). Recently, several studies have shown that $\mathrm{C}-\mathrm{C}$ motif chemokine receptor 7 (CCR7) plays an important role in the metastasis of head and neck SCC, esophageal carcinoma, gastric carcinoma, breast cancer, malignant melanoma, non-small cell lung carcinoma, and gastrointestinal carcinoma (9).

Neuropilin (NRP)1 and NRP2 are transmembrane glycoprotein receptors with roles in neuronal guidance, angiogenesis, and lymphangiogenesis via VEGF binding and signaling in the absence of other VEGF receptors (10). In addition, both are structural receptors of the class 3 semaphorins (11). Several studies have reported their expression and regulation in tumor cells of human breast, colon, and prostate cancers (7). NRP expression may be a prospective biomarker, and further studies may eventually lead to more efficacious treatments (7).

Semaphorin 3E (SEMA3E) is a secreted protein involved in axonal guidance and angiogenesis (12). There are eight groups of semaphorins, which bind to the cell surface receptors NRPs (NRP1 and NRP2) (13) as well as plexin-D1 for their signaling pathways (11). Casazza et al and other studies have reported that secreted SEMA3E and its receptor plexin-D1 inhibit tumor growth but promote the metastasis and invasiveness of cancer cells (14). They are involved in the metastasis of colon, liver, melanoma, and breast cancers; however, the mechanism remains unclear (13-15).

Although the predictive value of these biomarkers is yet to be established, it has been observed that lymphangiogenesis in cancer is not limited to the areas within or immediately adjacent to a primary tumor; however, it can also occur in the sentinel LNs $(16,17)$. The identification of effective and innovative therapies that appear to influence cancer metastasis is critical for the improvement of SCC therapy.

Therefore, the present study aimed to evaluate the expression levels of the following growth factors and receptors in SCC of the tongue: VEGF-C, VEGF-D, VEGFR-3, CCR7, NRP1, NRP2, and SEMA3E. In order to demonstrate the prognostic value of these proteins, we analyzed the correlation between them and the overall or disease-free survival. Furthermore, we assessed the correlation between microvessel density (MVD) and LN metastasis as well as the correlation between lymphatic vessel density (LVD) and LN metastasis, with respect to their clinicopathological features.

\section{Materials and methods}

Patients and tissues. All clinical studies were approved by the Ethics Committee of Osaka University Dental Hospital. We conducted a retrospective cohort study by randomly selecting 80 patients who had been previously diagnosed with primary tongue SCC and undergone curative tumor resection at the First Department of Oral and Maxillofacial Surgery, Osaka University Dental Hospital (Osaka, Japan) between 1995 and 2008.

Information regarding the clinicopathological features of each case (including age, gender, tumor size, nodal status, and the location and status of recurrence or metastasis) was obtained from the patient histories. The study included 55 men $(68.75 \%)$ and 25 women $(31.25 \%)$ between the ages of 22 and 92 years, with a median age of 62 years. Forty $(50 \%)$ of the patients had LN metastasis.
All patients were staged according to the 2010 Japan Society for Oral Tumors TNM Classification of Malignant Tumors (18). The histological mode of invasion was classified according to the YK classification $(19,20)$.

Immunohistochemistry. The paraffin sections were fixed in $10 \%$ neutral buffered formalin. Sections of $5-\mu \mathrm{m}$ thickness were cut consecutively, deparaffinized in xylene, rehydrated with graded concentrations of ethanol, and treated with citrate

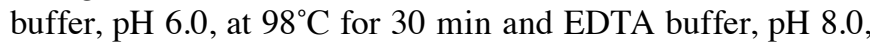
at $98^{\circ} \mathrm{C}$ for $15 \mathrm{~min}$ for heat-induced antigen retrieval. These sections were then used in histopathological and immunohistochemical analysis. To block endogenous peroxide activity, $0.3 \% \mathrm{H}_{2} \mathrm{O}_{2} \cdot \mathrm{dH}_{2} \mathrm{O}$ was applied to the sections. Non-specific reactions were blocked with $1 \%$ bovine serum albumin buffer [Histofine SAB-PO (Multi) kit; Nichirei Bioscience, Tokyo, Japan]. The sections were incubated with the following primary antibodies at $4^{\circ} \mathrm{C}$, overnight: anti-human rabbit polyclonal VEGF-C antibody (dilution 1:100), anti-human rabbit polyclonal VEGF-D antibody (dilution 1:200) (both from Abcam, Tokyo, Japan), anti-human mouse 9D9F9 VEGFR-3 antibody (dilution 1:500; Millipore Corp., Billerica, MA, USA), anti-human goat polyclonal CCR7 antibody (dilution 1:250), anti-human rabbit monoclonal NRP1 antibody (dilution 1:100) (both from Abcam), anti-human rabbit polyclonal NRP2 antibody (dilution 1:100; Atlas Antibodies, Stockholm, Sweden), anti-human goat polyclonal SEMA3E antibody (dilution 1:100; Abcam), anti-human mouse monoclonal CD-34 antibody (dilution 1:100), and anti-human mouse monoclonal D2-40 antibody (pre-diluted) (both from Nichirei Bioscience). Next, the appropriate secondary antibodies and blocking agents were applied using the Histofine SAB-PO (Multi or Goat) kit (Nichirei Bioscience). Immunostaining and immunolocalization of the proteins were performed using the DAB-Peroxidase Substrate Solution Immunohistochemistry brown Histofine SAB-PO (Multi) kit (Nichirei Bioscience), according to the manufacturer's instructions. The sections were counterstained with Mayer's hematoxylin solution (Sigma-Aldrich, St. Louis, MO, USA) dehydrated with graded concentrations of ethanol, cleared with xylene, and mounted for visualization using bright-field microscopy.

A positive control (immunohistochemical staining demonstrating weakly positive tissue) and negative controls (immunohistochemical staining with the omission of the primary antibody) were included in the staining protocol. Scoring and cell counts were performed without prior clinical knowledge of the patients.

Staining evaluation. The immunohistochemical staining of these proteins in SCC cells was evaluated based on the ratio and intensity of the staining. Specimens were considered immunopositive when $\geq 1 \%$ of the cancer cells showed clear evidence of immunostaining. The ratio of staining for each specimen was determined as follows: specimens with no positive tumor cells, score of $0 ; 1-25 \%$ positive cells, score of $1 ; 26-50 \%$ positive cells, score of $2 ; 51-75 \%$ positive cells, score of 3; and 76-100\% positive cells, score of 4 . The intensity of immunostaining was determined as follows: specimens with no staining, score of 0 ; weak staining, score of 1; moderate staining, score of 2; and intense staining, score 
A

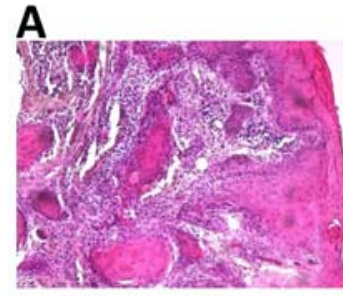

E

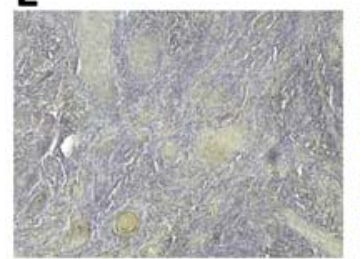

I

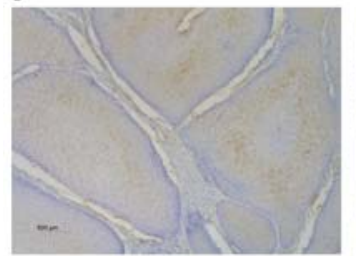

B

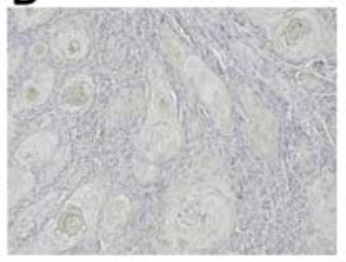

$\mathbf{F}$

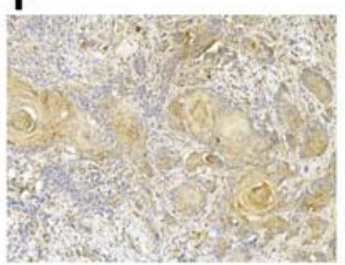

J

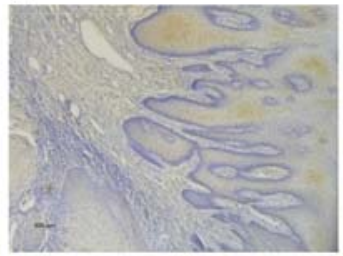

C

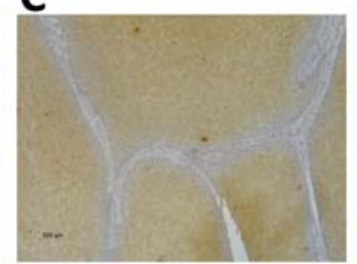

G
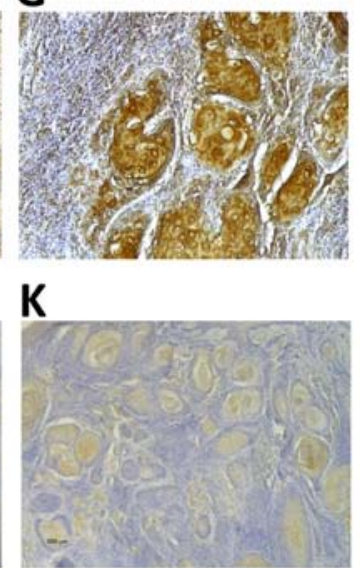

D

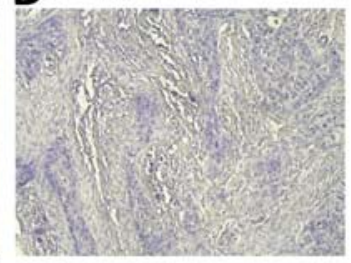

H

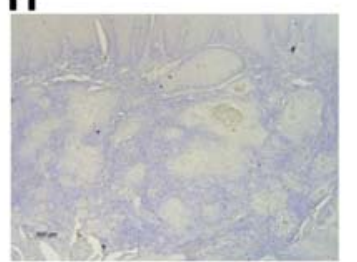

$\mathbf{L}$

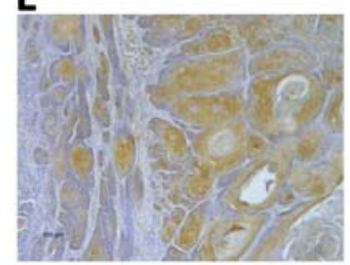

Figure 1. Immunohistochemical staining for biomarkers in SCC of the tongue. The expression of biomarkers in tongue SCC specimens, as determined by IHC staining, ranging from negative to weak, moderate, and strong. A score was given to each specimen according to the intensity of the staining and ratio of the positive tumor cells (x10 original magnification). (A) Hematoxylin and eosin staining of a SCC specimen. (B) Negative control IHC staining with omission of the primary antibody. (C) Positive control IHC staining for CCR7 demonstrates weakly positive tissue. (D) Negative IHC staining for CCR7 was given a score of 0 for intensity. (E) Weak IHC staining for CCR7 was given a score of 1 for intensity. (F) Moderate IHC staining for CCR7 was given a score of 2 for intensity. (G) Strong IHC staining for CCR7 was given a score of 3 for intensity. (H) Zero tumor cells positive for CCR7 was given a score of 0 for ratio. (I) Between 0 and $25 \%$ of the tumor cells positive for CCR7 staining was given a score of 1 for ratio. (J) Between 25 and $50 \%$ of the tumor cells positive for CCR7 staining were given a score of 2 for ratio. (K) Between 50 and $75 \%$ of the tumor cells positive for CCR7 staining was given a score of 3 for ratio. (L) Between 75 and $100 \%$ of the tumor cells positive for CCR7 staining were given a score of 4 for ratio. SCC, squamous cell carcinoma; IHC, immunohistochemical; CCR7, C-C motif chemokine receptor 7 .

of 3 (21-23) (Fig. 1). The tumors in this study were sometimes heterogeneous and the staining was therefore calculated by multiplying the ratio score with the intensity score to give an overall staining score between 1 and 12 , using a similar rationale to the immunoreactivity score (IRS) first described by Remmele and Stegner (24).

Using this scoring system, each component of the tumor was scored independently, and the results were calculated. The immunostaining of the specimens was evaluated independently by two authors (Dr Al-Shareef Hani and Dr Yosuke Shogen), who were blinded to patient results and other clinicopathological features, and their scores were averaged to obtain a final IRS. Subsequently, patients were divided into low and high expression groups, with the mean IRS for each protein serving as the cut-off value (21-23).

Assessment of MVD and LVD. MVD and LVD were estimated in tumor vessel 'hot spots' by immunostaining with the CD-34 and D2-40 podoplanin antibodies, respectively. Microvessel counts were assessed by light microscopy in areas of the tumor with the highest number of capillaries and small venules at the invasive edge, that is, in the areas with the most intense neovascularization. Although SCCs have a mostly heterogeneous MVD and LVD, areas of highly invasive carcinoma were identified by the presence of a higher number of discrete microvessels and lymphatic vessels that were positively stained for CD-34 and D2-40, respectively. These areas of increased neovascularization could occur anywhere in the invasive tumor; however, they were most frequently observed in the intratumoral region and at the margins of the carcinoma $(3,25)$.

Microvessels and lymphatic vessels with positive immunostaining for CD-34 and D2-40, respectively, were counted in three hotspots per section, using a 20x objective lens, by two authors (Dr Al-Shareef Hani and Dr Sanam Bakhshishayan), who were blinded to patient results and other clinicopathological features. The mean vessel counts in each of the three hotspots were recorded and each component of the tumor was scored independently to give six hotspot scores for each specimen. After averaging the three hotspot scores of each component, to obtain a final hotspot score for LVD and MVD, the specimens were divided into two groups (low or high) based on the mean values for LVD and MVD (6.85 and 20.31 number of vessels $/ \mu \mathrm{m}^{2}$ of tumor-free area, respectively). In brief, the area with the highest vascular density (hotspot) was selected, and the number of microvessels was determined in each of the three microscopic fields by each investigator using the 20x objective lens. The total lymphatic vessel count was compared between the groups according to age, gender, primary tumor site, clinical stage, tumor size, grade, and the status of the lymphatic metastasis.

Statistical analysis. Statistical analysis was performed using the statistical software IBM SPSS Advanced Statistics 20.0 (IBM Corp., Armonk, NY, USA). Subjects were grouped into 


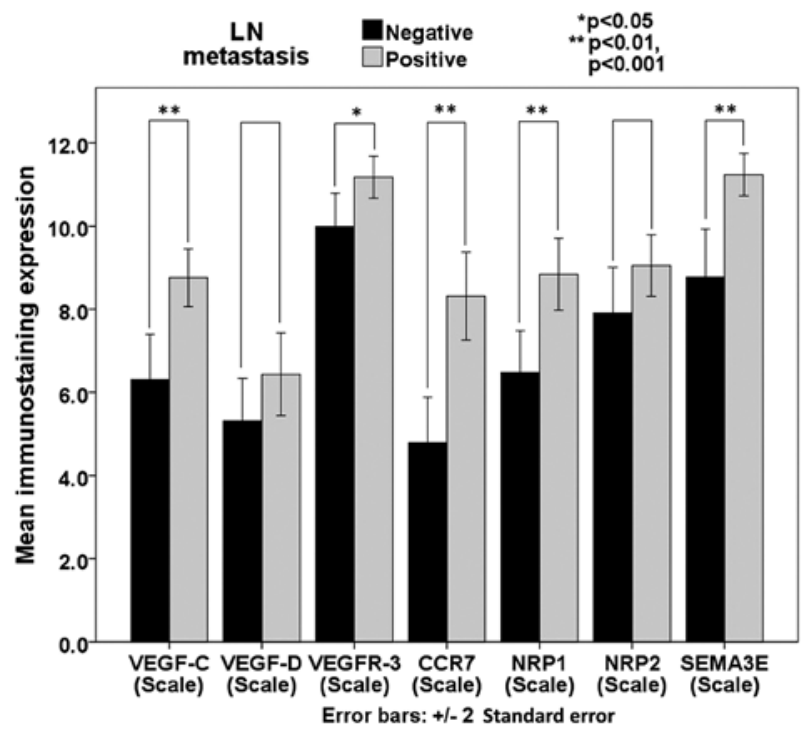

Figure 2. Relationship between the expression of proteins and LN metastasis. The relationship between protein expression and $\mathrm{LN}$ metastasis was analyzed by the independent samples t-test, and it is shown in this clustered bar chart. High expression levels for each of the proteins were identified in most of the specimens, and a significant association was demonstrated between LN metastasis and the expression levels of VEGF-C, VEGFR-3, CCR7, NRP1, and SEMA3E. ${ }^{*} \mathrm{P}<0.05 ;{ }^{* *} \mathrm{p}<0.01, \mathrm{p}<0.001$. LN, lymph node; VEGF, vascular endothelial growth factor; VEGFR-3, VEGF receptor-3; CCR7, C-C motif chemokine receptor 7; NRP, neuropilin; SEMA3E, semaphorin 3E.

the following categories: $<60$ or $\geq 60$ years of age; pathological negative (N0) or pathological positive (N1) for LN metastasis; and high or low protein expression based on the individual cut-off score for each protein. Chi-square and Fisher's exact tests were performed to assess the association between clinicopathological features and protein expression levels. An independent samples t-test was performed to determine the association between protein expression levels and LN metastasis. In addition, an independent samples t-test was performed to assess the relationship between LVD or MVD and LN metastasis or protein expression.

Univariate and multivariate logistic regression analyses were performed to assess the association between LN metastasis and clinicopathological features or protein expression as well as to assess the odds ratio (OR), and to define independent risk factors for prognosis and incidence of nodal recurrence. The overall and disease-free survival rates were estimated using the Kaplan-Meier method and analyzed using the log-rank test. For all tests, $\mathrm{p}<0.05$ was accepted as statistically significant.

\section{Results}

MVD, LVD, and the expression of angiogenic and lymphangiogenic biomarkers in oral SCC. The biomarkers for LN metastasis were analyzed individually. The expression levels of VEGF-C, VEGF-D, VEGFR-3, CCR7, NRP1, NRP2, and SEMA3E in oral SCC lesions were assessed by immunohistochemistry. We found that these biomarkers were predominantly expressed in the cell layer and the cytoplasm of the SCC cells, especially at the invasive edges, and were occasionally observed in endothelial cells in the stroma around or close to carcinoma nests and tumor vessels (Fig. 1).
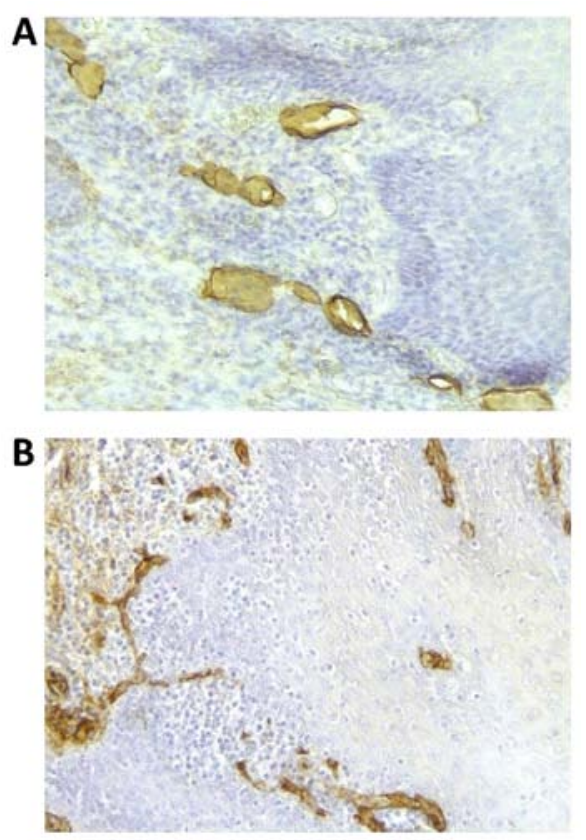

Figure 3. LVD and MVD evaluation. (A) Immunohistochemical analysis with the D2-40 podoplanin antibody was performed to evaluate the LVD. Positive staining for lymphatic vessels was detected at the invasive edge of the tumor (x20 original magnification). (B) Immunohistochemical analysis with the CD-34 antibody was performed to evaluate the blood MVD. Positive staining for microvessels was detected at the invasive edge of the tumor ( $\mathrm{x} 20$ original magnification). LVD, lymphatic vessel density; MVD, microvessel density.
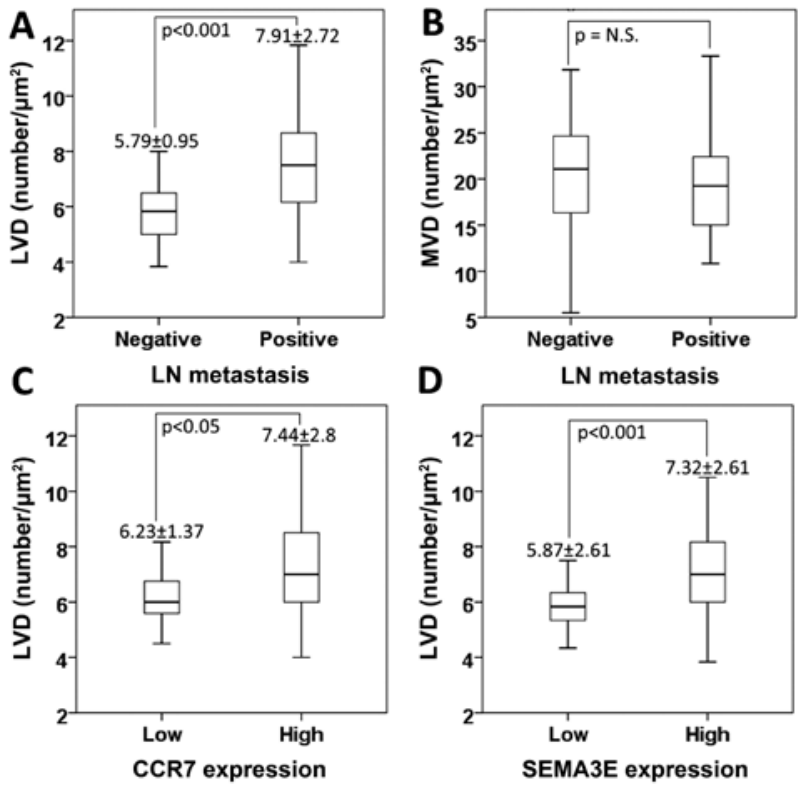

Figure 4. Relationship between predictive factors and MVD or LVD. The relationship between predictive factors and MVD or LVD was analyzed by the independent samples t-test, and it is shown in this box plot. (A) A significant correlation was shown between LVD and LN metastasis. (B) No association was observed between MVD and LN metastasis. (C) CCR7 expression showed a significant relationship with LVD. (D) SEMA3E expression showed a significant relationship with LVD. MVD, microvessel density; LVD, lymphatic vessel density; LN, lymph node; CCR7, C-C motif chemokine receptor 7; SEMA3E, semaphorin 3E; N.S., not significant.

High expression levels of VEGF-C, VEGF-D, VEGFR-3, CCR7, NRP1, NRP2, and SEMA3E were detected in 39 
Table I. Relationship between clinicopathological features and the expression levels of the proteins VEGF-C, VEGF-D, VEGFR-3, CCR7, NRP1, NRP2, and SEMA3E.

\begin{tabular}{|c|c|c|c|c|c|c|c|}
\hline & VEGF-C expression & VEGF-D ex & ression & VEG & R-3 ex & ression & CCR7 expression \\
\hline & P-value & High & $\mathrm{P}$-value & Low & High & P-value & High \\
\hline
\end{tabular}

\begin{tabular}{lrrrrrrrrrrrrr}
\hline Gender & & & & & & & & & & & & \\
$\quad$ Female & 25 & 14 & 11 & N.S. & 7 & 18 & 0.039 & 8 & 17 & 0.153 & 7 & 18 & 0.012 \\
$\quad$ Male & 55 & 27 & 28 & & 29 & 26 & & 27 & 28 & & 32 & 23 & \\
Age (years) & & & & & & & & & & & & & \\
$<60$ & 34 & 19 & 15 & N.S. & 20 & 14 & 0.033 & 21 & 13 & 0.005 & 19 & 15 & N.S. \\
$\geq 60$ & 46 & 22 & 24 & & 16 & 30 & & 14 & 32 & & 20 & 26 & \\
N classification & & & & & & & & & & & & & \\
N0 & 40 & 26 & 14 & 0.047 & 20 & 20 & N.S. & 24 & 16 & 0.035 & 27 & 13 & 0.015 \\
N1 & 13 & 4 & 9 & & 3 & 10 & & 5 & 8 & & 3 & 10 & \\
N2b & 18 & 7 & 11 & & 8 & 10 & & 5 & 13 & & 5 & 13 & \\
N2c & 7 & 2 & 5 & & 4 & 3 & & 1 & 6 & & 3 & 4 & \\
N3 & 2 & 2 & 0 & & 1 & 1 & & 0 & 2 & & 1 & 1 & \\
LN metastasis & & & & & & & & & & & & & \\
Negative & 40 & 26 & 14 & 0.014 & 20 & 20 & N.S. & 24 & 16 & 0.003 & 27 & 13 & 0.001 \\
Positive & 40 & 15 & 25 & & 16 & 24 & & 11 & 29 & & 12 & 28 & \\
\hline & & & & & & & & & & &
\end{tabular}

\begin{tabular}{|c|c|c|c|c|c|c|c|c|c|c|}
\hline & \multirow[b]{2}{*}{ Cases } & \multicolumn{3}{|c|}{ NRP1 expression } & \multicolumn{3}{|c|}{ NRP2 expression } & \multicolumn{3}{|c|}{ SEMA3E expression } \\
\hline & & Low & High & P-value & Low & High & P-value & Low & High & P-value \\
\hline \multicolumn{11}{|l|}{ Gender } \\
\hline Female & 25 & 10 & 15 & \multirow[t]{2}{*}{ N.S. } & 10 & 15 & \multirow[t]{2}{*}{ N.S. } & 7 & 18 & \multirow[t]{2}{*}{ N.S. } \\
\hline Male & 55 & 33 & 22 & & 25 & 30 & & 19 & 36 & \\
\hline \multicolumn{11}{|c|}{ Age (years) } \\
\hline$<60$ & 34 & 22 & 12 & \multirow[t]{2}{*}{ N.S. } & 17 & 17 & \multirow[t]{2}{*}{ N.S. } & 12 & 22 & \multirow[t]{2}{*}{ N.S. } \\
\hline$\geq 60$ & 46 & 21 & 25 & & 18 & 28 & & 14 & 32 & \\
\hline \multicolumn{11}{|c|}{$\mathrm{N}$ classification } \\
\hline No & 40 & 29 & 11 & \multirow[t]{5}{*}{0.012} & 20 & 20 & \multirow[t]{5}{*}{ N.S. } & 20 & 20 & \multirow[t]{5}{*}{0.016} \\
\hline N1 & 13 & 5 & 8 & & 3 & 10 & & 1 & 12 & \\
\hline $\mathrm{N} 2 \mathrm{~b}$ & 18 & 7 & 11 & & 8 & 10 & & 4 & 14 & \\
\hline $\mathrm{N} 2 \mathrm{c}$ & 7 & 1 & 6 & & 4 & 7 & & 1 & 6 & \\
\hline N3 & 2 & 1 & 1 & & 0 & 2 & & 0 & 2 & \\
\hline \multicolumn{11}{|c|}{ LN metastasis } \\
\hline Negative & 40 & 29 & 11 & \multirow[t]{2}{*}{0.001} & 20 & 20 & \multirow[t]{2}{*}{ N.S. } & 20 & 20 & \multirow[t]{2}{*}{0.001} \\
\hline Positive & 40 & 14 & 26 & & 15 & 25 & & 6 & 34 & \\
\hline
\end{tabular}

$\mathrm{p}<0.05, \mathrm{p}<0.01$ and $\mathrm{p}<0.001$ denote increasing levels of significance. VEGF, vascular endothelial growth factor; VEGFR-3, VEGF receptor-3; CCR7, C-C motif chemokine receptor 7; NRP, neuropilin; SEMA3E, semaphorin 3E; N.S., not significant; LN, lymph node.

(48.8\%), 44 (55\%), 45 (56.3\%), 41 (51.3\%), 37 (46.3\%), 45 $(56.3 \%)$, and $54(67.5 \%)$ tumor specimens, respectively. In contrast, 41 (51.3\%), 36 (45\%), 35 (43.8\%), 39 (48.8\%), 43 $(53.8 \%), 35(43.8 \%)$, and $26(32.5 \%)$ tumor specimens exhibited low expression levels of VEGF-C, VEGF-D, VEGFR-3, CCR7, NRP1, NRP2, and SEMA3E, respectively. The mean expression levels of VEGF-C, VEGF-D, VEGFR-3, CCR7, NRP1, NRP2, and SEMA3E were 7.538, 5.875, 10.581, 6.550, 7.663, 8.481, and 10.006, respectively (Fig. 2).
MVD was found to range between 5.5 and $38.5 \mu \mathrm{m}^{2}$ of blood vessels per square millimeter of the tumor (median, 19.92; mean \pm standard deviation (SD), 20.31 \pm 6.26 ), whereas LVD ranged between 3.83 and $20.83 \mu \mathrm{m}^{2}$ of lymphatic vessels per square millimeter of the tumor (median, 6.33; mean $\pm \mathrm{SD}$, 6.85 \pm 2.29 ) (Figs. 3 and 4).

The relationship between clinicopathological features and the expression of angiogenic and lymphangiogenic biomarkers. 
Chi-square and Fischer's exact tests were performed to assess the association between clinicopathological features and the expression levels of VEGF-C, VEGF-D, VEGFR-3, CCR7, NRP1, NRP2, and SEMA3E. The results showed that the expression level of VEGF-C was significantly associated with only pathological LN size $(\mathrm{p}<0.05)$ and LN metastasis $(\mathrm{p}<0.05)$, whereas the expression level of VEGF-D was significantly associated with only gender $(p<0.05)$ and age $(p<0.05)$. Moreover, the expression level of VEGFR-3 was significantly associated with only age $(\mathrm{p}<0.01), \mathrm{N}$ classification (Japan Society for Oral Tumors, classification for regional LN metastasis; $\mathrm{p}<0.05)$, and LN metastasis $(\mathrm{p}<0.01)$. The expression level of CCR7 was significantly associated only with gender $(\mathrm{p}<0.05), \mathrm{N}$ classification $(\mathrm{p}<0.05)$, and LN metastasis $(p=0.001)$. In addition, the expression level of NRP1 in the SCC cells was significantly associated only with pathological LN size $(\mathrm{p}<0.05)$ and $\mathrm{LN}$ metastasis $(\mathrm{p}=0.001)$, and the expression level of NRP2 was not significantly associated with any of the clinicopathological features, whereas the expression level of SEMA3E was significantly associated with only $\mathrm{N}$ classification $(\mathrm{p}<0.05)$ and LN metastasis $(\mathrm{p}=0.001)($ Table I).

The relationship between the expression levels of angiogenic and lymphangiogenic biomarkers and MVD or LVD. The relationship between angiogenesis and lymphangiogenesis in the primary SCC and LN metastasis was examined by assessing MVD, through immunohistochemical staining with the CD-34 antibody, and LVD, through immunohistochemical staining with the D2-40 antibody. LVD ranged between 3.83 and $20.83 \mu \mathrm{m}^{2}$ of lymphatic vessels per square millimeter of the tumor (median, 6.33; mean $\pm \mathrm{SD}, 6.85 \pm 2.29$ ). The independent samples t-test showed a significant association between LVD and LN metastasis, with the mean LVD being lower in the metastatic LN-negative group when compared to the metastatic LN-positive group [mean $\pm \mathrm{SD}, 5.79 \pm 0.95$ and $7.91 \pm 2.72$, respectively; $\mathrm{p}<0.001 ; \mathrm{t}(78)=4.66]$. No significant association was detected between MVD and LN metastasis.

The independent samples t-test was also used to assess the association between biomarker expression and MVD or LVD. We found that LVD was significantly associated with the expression of CCR7 $(\mathrm{p}<0.05)$ and SEMA3E $(\mathrm{p}<0.001)$, mainly at the invasive edges. However, no such association was detected between MVD and the expression of any of the biomarkers (Fig. 4) (Table II).

Logistic regression analysis of predictive factors and $L N$ metastasis. Univariate logistic regression analysis was performed to assess the association between clinicopathological features and LN metastasis. The results showed that SCC of cancer stage T4 is more likely to have $\mathrm{LN}$ metastasis (OR, 9.778; 95\% confidence interval (CI), 1.551-61.646; $\mathrm{p} \leq 0.05)$. Multivariate logistic regression analysis also showed that cancer stage T4 was significantly associated with LN metastasis (OR, 12.601; 95\% CI, 0.998-159.08; $\mathrm{p} \leq 0.05$ ). No significant relationship was identified between LN metastasis and the mode of invasion (YK classification), the differentiation of SCC cells, or other clinical factors in any of the regression analyses (Table III).

Univariate analysis showed significant association between LN metastasis and the expression levels of VEGF-C
Table II. The relationship between protein expression levels and MVD or LVD.

\begin{tabular}{|c|c|c|c|c|c|c|}
\hline & \multicolumn{3}{|c|}{ MVD } & \multicolumn{3}{|c|}{ LVD } \\
\hline & Mean & SD & P-value & Mean & SD & P-value \\
\hline \multicolumn{7}{|c|}{ LN metastasis } \\
\hline Negative & 20.79 & 6.72 & 0.497 & 5.79 & 0.95 & $<0.001$ \\
\hline Positive & 19.83 & 5.82 & & 7.91 & 2.72 & \\
\hline \multicolumn{7}{|l|}{ VEGF-C } \\
\hline Low & 21.28 & 5.49 & 0.154 & 6.53 & 1.5 & 0.154 \\
\hline High & 19.28 & 6.90 & & 7.18 & 2.89 & \\
\hline \multicolumn{7}{|l|}{ VEGF-D } \\
\hline Low & 20.17 & 6.19 & 0.856 & 6.72 & 1.54 & 0.207 \\
\hline High & 20.42 & 6.40 & & 6.95 & 2.77 & \\
\hline \multicolumn{7}{|l|}{ VEGFR-3 } \\
\hline Low & 19.71 & 6.17 & 0.458 & 6.46 & 1.46 & 0.183 \\
\hline High & 20.77 & 6.37 & & 7.15 & 2.75 & \\
\hline \multicolumn{7}{|l|}{ CCR7 } \\
\hline Low & 20.63 & 6.48 & 0.655 & 6.23 & 1.37 & 0.016 \\
\hline High & 20.00 & 6.11 & & 7.44 & 2.8 & \\
\hline \multicolumn{7}{|l|}{ NRP1 } \\
\hline Low & 20.33 & 6.27 & 0.979 & 6.42 & 1.62 & 0.69 \\
\hline High & 20.29 & 6.35 & & 7.35 & 2.83 & \\
\hline \multicolumn{7}{|l|}{ NRP2 } \\
\hline Low & 20.15 & 6.03 & 0.841 & 6.8 & 1.66 & 0.877 \\
\hline High & 20.43 & 6.50 & & 6.89 & 2.7 & \\
\hline \multicolumn{7}{|l|}{ SEMA3E } \\
\hline Low & 21.49 & 5.60 & 0.245 & 5.87 & 0.86 & $<0.001$ \\
\hline High & 19.74 & 6.53 & & 7.32 & 2.61 & \\
\hline
\end{tabular}

$\mathrm{p}<0.05, \mathrm{p}<0.01$ and $\mathrm{p}<0.001$ denote levels of significance. MVD, microvessel density; LVD, lymphatic vessel density; SD, standard deviation; LN, lymph node; VEGF, vascular endothelial growth factor; VEGFR-3, VEGF receptor-3; CCR7, C-C motif chemokine receptor 7; NRP, neuropilin; SEMA3E, semaphorin 3E.

(OR, 3.095; 95\% CI, 1.243-7.706; p<0.05), VEGFR-3 (OR, 3.955; 95\% CI, 1.546-10.114; p<0.01), CCR7 (OR, 4.846; 95\% CI, 1.882-12.482; p=0.001), NRP1 (OR, 4.896; 95\% CI, 1.892-12.669; $\mathrm{p}=0.001$ ), and SEMA3E (OR, 5.667; $95 \% \mathrm{CI}, 1.951-16.462 ; \mathrm{p}=0.001)$. No correlation was identified between LN metastasis and the expression levels of NRP2 and VEGF-D. However, a significant association was observed between LN metastasis and LVD (OR, 2.832; 95\% CI, 1.716-4.673; p<0.0001), but not MVD. By contrast, multivariate analysis showed only the association between LN metastasis and NRP1 expression level (OR, 5.905; 95\% CI, 1.274-27.372; $\mathrm{p}<0.05$ ) or LVD (OR, 2.527; 95\% CI, 1.412-4.522; p<0.01). No correlation was identified between LN metastasis and the expression levels of the other proteins (Table III).

The relationship between the expression of angiogenic and lymphangiogenic biomarkers and survival time. We 
Table III. Relationship between the predictive factors and LN metastasis.

\begin{tabular}{|c|c|c|c|c|c|c|}
\hline & \multicolumn{3}{|c|}{ Univariate } & \multicolumn{3}{|c|}{ Multivariate } \\
\hline & Odds ratio & $95 \% \mathrm{CI}$ & P-value & Odds ratio & $95 \% \mathrm{CI}$ & P-value \\
\hline $\mathrm{T}$ stage $\mathrm{T} 1 \mathrm{ref} \mathrm{T} 4$ & 9.778 & $1.551-61.646$ & 0.015 & 12.601 & $0.998-159.08$ & 0.05 \\
\hline VEGF-C expression & 3.095 & $1.243-7.706$ & 0.015 & 2.06 & $0.462-9.178$ & 0.343 \\
\hline VEGFR-3 expression & 3.955 & $1.546-10.114$ & 0.004 & 2.436 & $0.484-12.272$ & 0.28 \\
\hline CCR7 expression & 4.846 & $1.882-12.482$ & 0.001 & 1.402 & $0.301-6.538$ & 0.667 \\
\hline NRP1 expression & 4.896 & $1.892-12.669$ & 0.001 & 5.905 & $1.274-27.372$ & 0.023 \\
\hline SEMA3E expression & 5.667 & $1.951-16.462$ & 0.001 & 1.979 & $0.389-10.065$ & 0.411 \\
\hline LVD (D2-40) & 2.832 & $1.716-4.673$ & $<0.001$ & 2.527 & $1.412-4.522$ & 0.002 \\
\hline
\end{tabular}

$\mathrm{p} \leq 0.05, \mathrm{p}<0.01$ and $\mathrm{p}<0.001$ denote increasing levels of significance. LN, lymph node; CI, confidence interval; VEGF, vascular endothelial growth factor; VEGFR-3, VEGF receptor-3; CCR7, C-C motif chemokine receptor 7; NRP, neuropilin; SEMA3E, semaphorin 3E; LVD, lymphatic vessel density.

used the Kaplan-Meier method to determine the association between $\mathrm{LN}$ metastasis and prognosis in patients with oral SCC. The results showed a reduced overall survival time for patients with metastatic SCC when compared to patients with non-metastatic cancer (patients with LN metastasis were associated with overall survival time and LVD exhibited an association, log-rank $\mathrm{p}<0.001$ ) (Fig. 5).

The Kaplan-Meier analysis also showed that patients with high expression levels of VEGF-C, VEGFR-3, NRP1, and SEMA3E, were more likely to have localized or regional recurrence after treatment (VEGF-C, VEGFR-3, NRP1, and SEMA3E were associated with disease-free survival time, $\log$-rank $\mathrm{p}<0.05)$.

Patients with metastatic SCC were also more likely to have localized or regional recurrence after treatment (patients with $\mathrm{LN}$ metastasis were associated with disease-free survival time and LVD exhibited an association, log-rank p<0.001) (Fig. 5). To summarize, $\mathrm{LN}$ metastasis was found to be associated with poor survival in SCC.

\section{Discussion}

In the present study, several biomarkers have been proposed for SCC. The aim of this study was to evaluate the expression levels of the following growth factors and receptors in SCC of the tongue: VEGF-C, VEGF-D, VEGFR-3, CCR7, NRP1, NRP2, and SEMA3E. Furthermore, we investigated the association between the expression levels of these proteins and intratumoral MVD, using CD-34 antibody, or LVD, using D2-40 podoplanin antibody, as indicators of angiogenesis and lymphangiogenesis, respectively.

The PI3K/AKT, ERK1/2, and p38 pathways are crucial for cellular proliferation and survival (26). However, the association between disease-specific survival and the expression of VEGF-C, VEGF-D, and their receptor VEGFR-3 remains controversial. Many studies have proposed a strong association between LN metastasis and the expression levels of VEGF-C and VEGF-D in SCC. However, the association between LN metastasis and VEGF-D is controversial and has not been supported by other studies. In fact, VEGF-D has been reported to act as a suppressor in some types of cancers $(27,28)$. A relationship between $\mathrm{LN}$ metastasis and the expression levels of VEGF-C or VEGFR-3 has been reported for head and neck SCC, gastric, breast, thyroid, prostate, esophageal, and colorectal carcinomas (6).

The present study showed that LN metastasis is significantly correlated with the expression levels of VEGF-C and VEGFR-3, but not VEGF-D, based on immunohistochemical staining $(\mathrm{p}<0.05)$ and univariate logistic regression analysis $(\mathrm{p}<0.01)$. Immunostaining for these proteins was extensively associated with some clinicopathological features and was strongly correlated with disease-specific survival, suggesting a role for VEGF-C and VEGFR-3 as biomarkers for oral SCC metastasis. These results are in agreement with other reports on head and neck cancer (29), which suggest that VEGF-D is less important than VEGF-C for LN metastasis. However, it is still necessary for the metastasis mechanism, although its role is presently controversial. The possibility that VEGFR-3 expression in cancer cells could be associated with increased regional $\mathrm{LN}$ metastasis in oral $\mathrm{SCC}$ is also in agreement with other recent studies, suggesting a role for VEGFR-3 expression in predicting $\mathrm{LN}$ metastasis of SCC.

Furthermore, we found a strong relationship between the expression levels of both VEGF-C and VEGFR-3 and lymphangiogenesis in patients with oral SCC. Many clinical and preclinical studies have suggested that clinicopathological features are usually unreliable predictors of $\mathrm{LN}$ metastasis, with highly variable results, particularly for oral SCC (30-32). However, in this study, we suggest that LVD could be a predictive marker for LN metastasis in oral SCC. Furthermore, VEGF-C/VEGFR-3 pathway inhibition has been shown to inhibit cancer progression (33-36). Therefore, signaling through VEGFR-3 is not necessarily a result of LN metastasis. The fact that VEGFR-3 can form heterodimers with VEGFR-2 makes this mechanism even more complicated (17).

This study also predicted a strong correlation between LVD and LN metastasis in oral SCC. Both disease-free and overall 

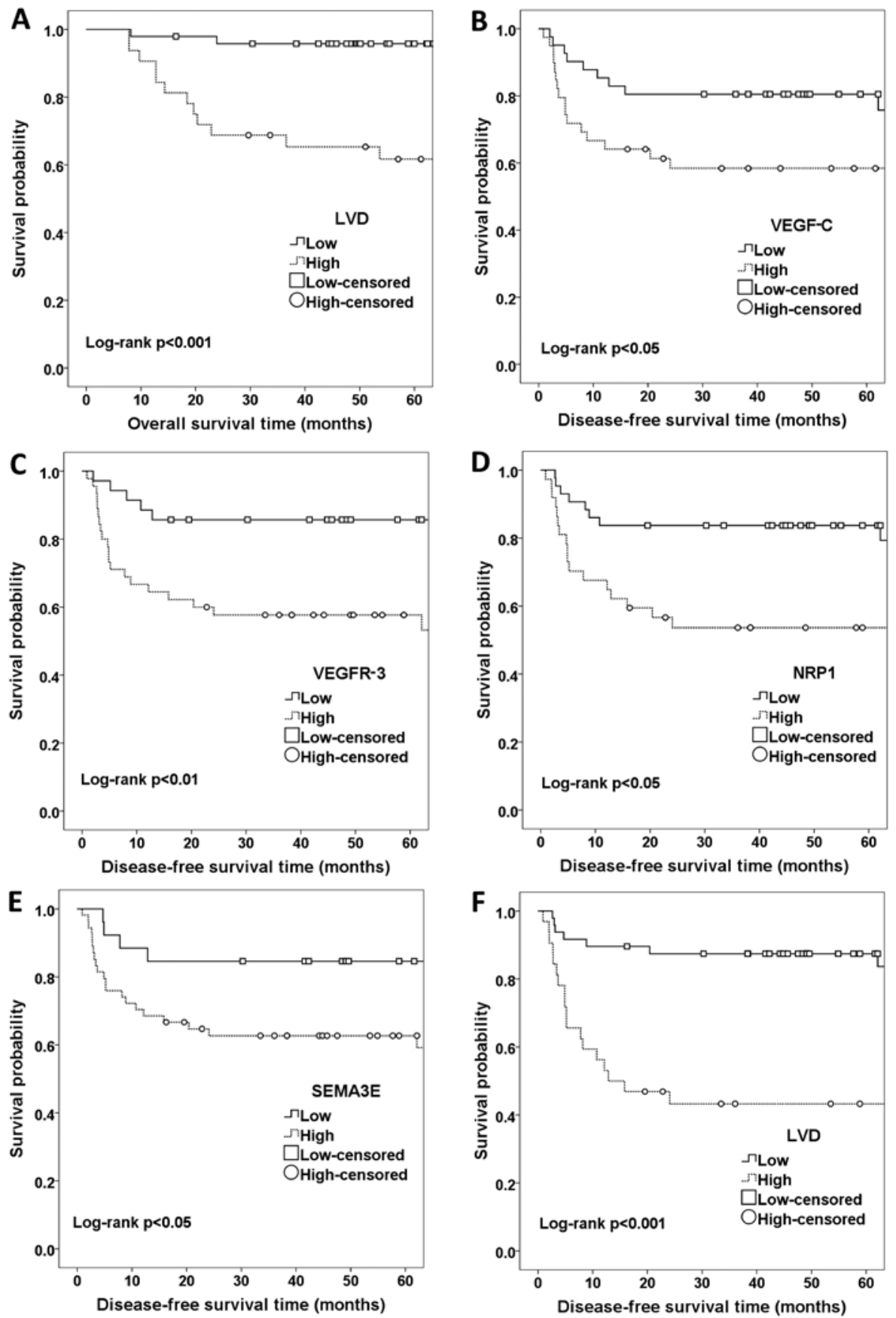

Figure 5. The association between predictive factors and patient survival or prognosis. The association between predictive factors and patient survival was measured using the Kaplan-Meier method. LVD showed an association with (A) the overall survival time as well as (F) the disease-free survival time, whereas (B) VEGF-C, (C) VEGFR-3 , (D) NRP1 and (E) SEMA3E showed an association with disease-free survival time. LVD, lymphatic vessel density; NRP, neuropilin; VEGF, vascular endothelial growth factor; VEGFR-3, VEGF receptor-3; SEMA3E, semaphorin 3E.

survival were associated with intra- and peritumoral LVD, as patients with a high LVD were revealed to have poor survival and a high possibility of recurrence. We did not detect any association between MVD and LN metastasis or prognosis. Other studies, by systematic review, have shown that MVD is unlikely to be a prognostic factor in early stage non-small cell lung cancer patients (6).

The association of angiogenic and lymphangiogenic biomarkers with prognosis in SCC patients remains controversial due to the variable outcomes reported by independent studies $(17,37)$. Some biomarkers have been correlated with poor prognosis or prolonged survival (38-40), whereas others have failed to show any prognostic significance (37). In our study, VEGF-C and VEGFR-3 expression levels were correlated with poor prognosis and a high possibility of localized or regional recurrence after treatment.

Many studies have reported that the expression of CCR7 in tumors, such as metastatic tumor cells of gastric, esophageal, breast, renal, oropharyngeal, and head and neck cancers, is significantly correlated with poor prognosis. Many studies have also reported a relationship between CCR7 expression levels and oral as well as head and neck SCC $(9,41)$. 
In the present study, we found that CCR7 immunostaining was strong in tongue cancer tissue. Univariate logistic regression analysis showed that CCR7 expression was significantly correlated with LN metastasis $(\mathrm{p}=0.001)$ as well as some clinicopathological features. Furthermore, a significant association was detected between upregulated CCR7 expression and LVD; however, no association was estimated between this upregulated CCR7 expression and prognosis. The results showed a possible role for CCR7 in the progression of tongue SCC as well as in mediating signaling in metastatic SCC.

The interactions between the proteins in the VEGF pathway are complex; which includes VEGF-A, VEGF-B, VEGF-C, VEGF-D, and PIGF, their tyrosine kinase receptors VEGFR-1, VEGFR-2, and VEGFR-3, and two NRP receptors NRP1 and NRP2. The VEGF receptors can form complexes with the NRP receptors and modulate the signaling outcomes through p38 MAPK pathway activation, leading to migration and survival of endothelial cells. Semaphorin family competes with VEGFs to bind to NRPs and modulate the angiogenesis mechanism (42). Many previous studies have investigated the expression of NRP1 and NRP2 in SCC of the tongue, esophagus, colon/rectum, breast, stomach, and lungs (10). Moreover, some studies have shown that NRP1 had a higher expression level compared to that of NRP2 (7). NRP1 and NRP2 expression levels have been shown to be prospective indicators of poor prognosis in colorectal cancer (7). However, other studies have found that NRP1 was neither correlated with any clinical features nor with poor prognosis in SCC of the tongue (43).

In the present study, NRP1 was shown, by immunohistochemical analysis, to be extensively expressed in cancerous tongue tissue. Univariate logistic regression analysis showed that NRP1 expression was significantly correlated with LN metastasis $(p=0.001)$ as well as with some other clinicopathological features (such as $\mathrm{N}$ classification). This indicates the controversial role of NRP1 in tumor growth and progression. The upregulation of NRP1 was also significantly correlated with poor disease-free survival. However, NRP2 did not show any association with $\mathrm{LN}$ metastasis, prognosis, or clinicopathological features. A possible explanation may be that NRP1 inhibits apoptosis by binding to VEGF and NRP2 increases the epithelial-mesenchymal transition (7). Furthermore, NRP1 expression appears to be more involved in angiogenesis and lymphangiogenesis than NRP2 expression. In general, these data suggest that both NRP1 and NRP2 are possible targets for chemotherapy; however, agents targeting NRP1 may have a greater anti-angiogenic effects in SCC.

Semaphorins, and their receptors, are a large family of extracellular signaling proteins with important roles in angiogenesis and tumor progression. SEMA3E is a secreted protein that recognizes the plexin-D1 receptor as well as the NRP receptors $(12,44)$. However, the functional relevance of SEMA3E in cancer is poorly understood. In addition to providing inhibitory signals for angiogenesis, SEMA3E/plexin-D1 signaling can regulate other cell types $(42,45)$. Casazza et al found that 'SEMA3E inhibits tumor growth, but promotes metastasis', in an NRP-independent manner (14).
SEMA3E expression is positively correlated with metastatic progression in highly invasive and metastatic mammary carcinomas, high- vs. low-grade glioblastomas, colon and liver cancers, and melanomas $(13,14)$. Increased expression of SEMA3E has been shown in metastatic cancers when compared to that of non-metastatic cancers. Kaplan-Meier survival analysis revealed that SEMA3E expression levels in primary tumors were significantly inversely correlated with patient survival, whereas the opposite was true for bladder carcinomas (14) as well as for primary and metastatic prostate tumors (42).

The present study is the first time where SEMA3E expression has been investigated in SCC of the tongue. Univariate logistic regression analysis revealed that SEMA3E expression was significantly correlated with LN metastasis $(p=0.001)$. SEMA3E was shown to be highly expressed in immunohistochemical analysis, and its association with clinicopathological features was stronger than the association detected between upregulated CCR7 expression and LVD. In addition, SEMA3E is strongly correlated with disease-specific survival, suggesting its importance as a biomarker in oral SCC metastasis. This result is consistent with previous studies in colon and liver cancers, melanomas, and metastatic mammary carcinomas. The finding that SEMA3E is also important in tongue SCC suggests that its signaling is crucially involved in the metastatic mechanisms of multiple cancer cells and is therefore a promising therapeutic target for the prevention of tumor metastasis.

Due to the limitations of this retrospective cohort study, the analysis of archival documents is a better way to analyze multiple outcomes and deal with potential heterogeneity and rare cases. This may also help in obtaining sufficient data for the identification of prognostic and predictive biomarkers and may improve the study design of randomized trials. Further studies in larger cohorts would better validate our results for oral SCC patients.

In summary, we suggest that high expression levels of VEGF-C, VEGFR-3, CCR7, and SEMA3E, as detected by immunohistochemical staining, serve as non-independent predictors of LN metastasis. Furthermore, LVD and NRP1 were found to be independent predictors of LN metastasis in SCC of the tongue. Statistical regression analysis and prognostic analysis showed that these factors are more likely to predict LN metastasis; therefore, these factors would be useful in estimating the possibility of LN metastasis in SCC. The accurate assessment of $\mathrm{LN}$ metastasis is crucial to improve the survival of oral cancer patients after treatment.

\section{Acknowledgements}

This study was supported by grants from the Ministry of Education, Culture, Sports, Science, and Technology in Japan and by JSPS KAKENHI (grant no. 15K20519).

\section{References}

1. Lee D, Wada K, Taniguchi Y, Al-Shareef H, Masuda T, Usami Y, Aikawa T, Okura M, Kamisaki Y and Kogo M: Expression of fatty acid binding protein 4 is involved in the cell growth of oral squamous cell carcinoma. Oncol Rep 31: 1116-1120, 2014. 
2. Doshi NP, Shah SA, Patel KB and Jhabuawala MF: Histological grading of oral cancer: A comparison of different systems and their relation to lymph node metastasis. Nat J Commun Med 2: 136-142, 2011.

3. Watanabe S, Kato M, Kotani I, Ryoke K and Hayashi K: Lymphatic vessel density and vascular endothelial growth factor expression in squamous cell carcinomas of lip and oral cavity: A clinicopathological analysis with immunohistochemistry using antibodies to D2-40, VEGF-C and VEGF-D. Yonago Acta Med 56: 29-37, 2013.

4. Miyata Y, Kanda S, Ohba K, Nomata K, Hayashida Y, Eguchi J, Hayashi T and Kanetake H: Lymphangiogenesis and angiogenesis in bladder cancer: Prognostic implications and regulation by vascular endothelial growth factors-A, -C, and -D. Clin Cancer Res 12: 800-806, 2006.

5. Hicklin DJ and Ellis LM: Role of the vascular endothelial growth factor pathway in tumor growth and angiogenesis. J Clin Oncol 23: 1011-1027, 2005.

6. Sugiura T, Inoue Y, Matsuki R, Ishii K, Takahashi M, Abe M and Shirasuna K: VEGF-C and VEGF-D expression is correlated with lymphatic vessel density and lymph node metastasis in oral squamous cell carcinoma: Implications for use as a prognostic marker. Int J Oncol 34: 673-680, 2009.

7. Staton CA, Koay I, Wu JM, Hoh L, Reed MW and Brown NJ: Neuropilin-1 and neuropilin-2 expression in the adenoma-carcinoma sequence of colorectal cancer. Histopathology 62 : 908-915, 2013

8. Kodama J, Hasengaowa, Kusumoto T, Seki N, Matsuo T, Ojima Y, Nakamura K, Hongo A and Hiramatsu Y: Association of CXCR4 and CCR7 chemokine receptor expression and lymph node metastasis in human cervical cancer. Ann Oncol 18: 70-76, 2007.

9. Ishida K, Iwahashi M, Nakamori M, Nakamura M, Yokoyama S Iida T, Naka T, Nakamura Y and Yamaue H: High CCR7 mRNA expression of cancer cells is associated with lymph node involvement in patients with esophageal squamous cell carcinoma. Int J Oncol 34: 915-922, 2009.

10. Alattar M, Omo A, Elsharawy M and Li J: Neuropilin-1 expression in squamous cell carcinoma of the oesophagus. Eur J Cardiothorac Surg 45: 514-520, 2014.

11. Nasarre P, Gemmill RM and Drabkin HA: The emerging role of class-3 semaphorins and their neuropilin receptors in oncology. Onco Targets Ther 7: 1663-1687, 2014.

12. Kumanogoh A and Kikutani H: Immunological functions of the neuropilins and plexins as receptors for semaphorins. Nat Rev Immunol 13: 802-814, 2013.

13. Klagsbrun M and Shimizu A: Semaphorin 3E, an exception to the rule. J Clin Invest 120: 2658-2660, 2010.

14. Casazza A, Finisguerra V, Capparuccia L, Camperi A, Swiercz JM, Rizzolio S, Rolny C, Christensen C, Bertotti A, Sarotto I, et al: Sema3E-Plexin D1 signaling drives human cancer cell invasiveness and metastatic spreading in mice. J Clin Invest 120: 2684-2698, 2010.

15. Luchino J, Hocine M, Amoureux MC, Gibert B, Bernet A Royet A, Treilleux I, Lécine P, Borg JP, Mehlen P, et al: Semaphorin 3E suppresses tumor cell death triggered by the plexin D1 dependence receptor in metastatic breast cancers. Cancer Cell 24: 673-685,2013.

16. Franceschi S, Bidoli E, Herrero R and Muñoz N: Comparison of cancers of the oral cavity and pharynx worldwide: Etiological clues. Oral Oncol 36: 106-115, 2000.

17. Anagnostou VK, Tiniakos DG, Fotinou M, Achimastos A and Syrigos KN: Multiplexed analysis of angiogenesis and lymphangiogenesis factors predicts outcome for non-small cell lung cancer patients. Virchows Arch 458: 331-340, 2011.

18. Izumo T, Kirita T, Ariji E, Ozeki S, Okada N, Okabe S Okazaki Y, Omura K, Kusama M, Sato T, et al; Working Group 1 on the 'Guidelines for Clinical and Pathological Studies of Oral Cancer', Scientific Committee, Japan Society for Oral Tumors: General rules for clinical and pathological studies on oral cancer: A synopsis. Jpn J Clin Oncol 42: 1099-1109, 2012.

19. Yamamoto E, Kohama G, Sunakawa H, Iwai M and Hiratsuka H: Mode of invasion, bleomycin sensitivity, and clinical course in squamous cell carcinoma of the oral cavity. Cancer 51: 2175-2180, 1983.

20. Yamamoto E, Miyakawa A and Kohama G: Mode of invasion and lymph node metastasis in squamous cell carcinoma of the oral cavity. Head Neck Surg 6: 938-947, 1984.

21. Barnes DM, Harris WH, Smith P, Millis RR and Rubens RD: Immunohistochemical determination of oestrogen receptor: Comparison of different methods of assessment of staining and correlation with clinical outcome of breast cancer patients. Br J Cancer 74: 1445-1451, 1996.
22. Ding Y, Shimada Y, Maeda M, Kawabe A, Kaganoi J, Komoto I, Hashimoto $Y$, Miyake $M$, Hashida $H$ and Imamura $M$ : Association of $\mathrm{CC}$ chemokine receptor 7 with lymph node metastasis of esophageal squamous cell carcinoma. Clin Cancer Res 9: 3406-3412, 2003

23. Krajewska M, Krajewski S, Epstein JI, Shabaik A, Sauvageot J, Song K, Kitada S and Reed JC: Immunohistochemical analysis of bcl-2, bax, bcl-X, and mcl-1 expression in prostate cancers. Am J Pathol 148: 1567-1576, 1996.

24. Remmele $\mathrm{W}$ and Stegner HE: Immunhistochemischer nachweis von östrogenerezeptoren (ER-ICA) im mammakarizi- nomgewebe: Vorschlag zur einheitlichen bewertung des untersu- chungsbefundes. Frauenarzt 28: 41-43, 1987.

25. Weidner N, Semple J, Welch W and Folkman J: Tumor angiogenesis and metastasis - correlation in invasive breast carcinoma. N Engl J Med 324: 1-8, 1991.

26. Feng Y, Hu J, Ma J, Feng K, Zhang X, Yang S, Wang W, Zhang $J$ and Zhang Y: RNAi-mediated silencing of VEGF-C inhibits non-small cell lung cancer progression by simultaneously down-regulating the CXCR4, CCR7, VEGFR-2 and VEGFR-3-dependent axes-induced ERK, p38 and AKT signalling pathways. Eur J Cancer 47: 2353-2363, 2011.

27. Niki T, Iba S, Tokunou M, Yamada T, Matsuno Y and Hirohashi S: Expression of vascular endothelial growth factors A, B, C, and D and their relationships to lymph node status in lung adenocarcinoma. Clin Cancer Res 6: 2431-2439, 2000

28. George ML, Tutton MG, Janssen F, Arnaout A, Abulafi AM, Eccles SA and Swift RI: VEGF-A, VEGF-C, and VEGF-D in colorectal cancer progression. Neoplasia 3: 420-427, 2001.

29. Shintani S, Li C, Ishikawa T, Mihara M, Nakashiro K and Hamakawa $\mathrm{H}$ : Expression of vascular endothelial growth factor A, B, C, and D in oral squamous cell carcinoma. Oral Oncol 40: 13-20, 2004.

30. Kitadai Y,Amioka T,Haruma K, Tanaka S, Yoshihara M,Sumii K, Matsutani N, Yasui W and Chayama K: Clinicopathological significance of vascular endothelial growth factor (VEGF)-C in human esophageal squamous cell carcinomas. Int J Cancer 93: 662-666, 2001

31. Lalla RV, Boisoneau DS, Spiro JD and Kreutzer DL: Expression of vascular endothelial growth factor receptors on tumor cells in head and neck squamous cell carcinoma. Arch Otolaryngol Head Neck Surg 129: 882-888, 2003.

32. Sanmartín E, Sirera R, Usó M, Blasco A, Gallach S, Figueroa S, Martínez N, Hernando C, Honguero A, Martorell M, et al: A gene signature combining the tissue expression of three angiogenic factors is a prognostic marker in early-stage non-small cell lung cancer. Ann Surg Oncol 21: 612-620, 2014.

33. Iljin K, Karkkainen MJ, Lawrence EC, Kimak MA, Uutela M, Taipale J, Pajusola K, Alhonen L, Halmekytö M, Finegold DN, et al: VEGFR3 gene structure, regulatory region, and sequence polymorphisms. FASEB J 15: 1028-1036, 2001.

34. Neuchrist C, Erovic BM, Handisurya A, Fischer MB, Steiner GE, Hollemann D, Gedlicka C, Saaristo A and Burian M: Vascular endothelial growth factor $\mathrm{C}$ and vascular endothelial growth factor receptor 3 expression in squamous cell carcinomas of the head and neck. Head Neck 25: 464-474, 2003.

35. Su JL, Yang PC, Shih JY, Yang CY, Wei LH, Hsieh CY, Chou CH, Jeng YM, Wang MY, Chang KJ, et al: The VEGF-C/Flt-4 axis promotes invasion and metastasis of cancer cells. Cancer Cell 9: 209-223, 2006

36. Chaudary N, Milosevic M and Hill RP: Suppression of vascular endothelial growth factor receptor 3 (VEGFR3) and vascular endothelial growth factor C (VEGFC) inhibits hypoxia-induced lymph node metastases in cervix cancer. Gynecol Oncol 123: 393-400, 2011

37. Zhan P, Wang J, Lv XJ, Wang Q, Qiu LX, Lin XQ, Yu LK and Song Y: Prognostic value of vascular endothelial growth factor expression in patients with lung cancer: A systematic review with meta-analysis. J Thorac Oncol 4: 1094-1103, 2009.

38. Carrillo de Santa Pau E, Arias FC, Caso Peláez E, Muñoz Molina GM, Sánchez Hernández I, Muguruza Trueba I, Moreno Balsalobre R, Sacristán López S, Gómez Pinillos A and del Val Toledo Lobo M: Prognostic significance of the expression of vascular endothelial growth factors A, B, C, and D and their receptors $\mathrm{R} 1, \mathrm{R} 2$, and $\mathrm{R} 3$ in patients with nonsmall cell lung cancer. Cancer 115: 1701-1712, 2009.

39. Maekawa S, Iwasaki A, Shirakusa T, Enatsu S, Kawakami T, Kuroki $\mathrm{M}$ and Kuroki M: Correlation between lymph node metastasis and the expression of VEGF-C, VEGF-D and VEGFR-3 in T1 lung adenocarcinoma. Anticancer Res 27: 3735-3741, 2007. 
40. Saintigny P, Kambouchner M, Ly M, Gomes N, SainteCatherine O, Vassy R, Czernichow S, Letoumelin P, Breau JL, Bernaudin JF, et al: Vascular endothelial growth factor-C and its receptor VEGFR-3 in non-small-cell lung cancer: Concurrent expression in cancer cells from primary tumour and metastatic lymph node. Lung Cancer 58: 205-213, 2007.

41. Pitkin L, Luangdilok S, Corbishley C, Wilson POG, Dalton P, Bray D, Mady S, Williamson P, Odutoye T, Rhys Evans P, et al: Expression of CC chemokine receptor 7 in tonsillar cancer predicts cervical nodal metastasis, systemic relapse and survival. Br J Cancer 97: 670-677, 2007.

42. Bender RJ and Mac Gabhann F: Dysregulation of the vascular endothelial growth factor and semaphorin ligand-receptor families in prostate cancer metastasis. BMC Syst Biol 9: 55, 2015.
43. Song X, Zhang W, Zhang Y, Zhang H, Fu Z, Ye J, Liu L, Song X and Wu Y: Expression of semaphorin $3 \mathrm{~A}$ and neuropilin 1 with clinicopathological features and survival in human tongue cancer. Med Oral Patol Oral Cir Bucal 17: e962-e968, 2012.

44. Cagnoni G and Tamagnone L: Semaphorin receptors meet receptor tyrosine kinases on the way of tumor progression. Oncogene 33: 4795-4802, 2014.

45. Tamagnone L and Rehman M: To die or not to die: Sema3E rules the game. Cancer Cell 24: 564-566, 2013. 